\title{
Large Eddy Simulation of Fire Dynamics with the Improved Eddy Dissipation Concept
}

\author{
ZHIBIN CHEN, JENNIFER WEN, BAOPENG XU, and SIAKA DEMBELE \\ Centre for Fire and Explosion Studies \\ Kingston University \\ Friars Avenue, Roehampton Vale, London, SW15 3DW, UK
}

\begin{abstract}
The eddy dissipation concept (EDC) for turbulent combustion modeling was originally proposed by Magnussen in the Reynolds averaged Navier-Stokes (RANS) context. This study has extended it to the large eddy simulation (LES) framework. Since the fine structures in EDC are still not resolved in LES, they are modeled with SGS turbulent kinetic energy and its dissipation rate instead of the mean quantities in RANS. A new expression is proposed for the reacting fraction of the fine structures to alleviate the limitations of the original formula. Three fire cases including a $7.1 \mathrm{~cm}$ methane fire, a $30 \mathrm{~cm}$ heptane fire and a $30.5 \mathrm{~cm}$ methanol fire are simulated to verify the improved EDC (M-EDC), which has been implemented in the FireFOAM solver. The predictions from the M-EDC are found to be in reasonably good agreement with the measurements, while the original EDC tends to under-predict temperature and velocity.
\end{abstract}

KEYWORDS: CFD, EDC, combustion, large eddy simulation, pool fires.

\section{NOMENCLATURE LISTING}

\begin{tabular}{|c|c|c|c|}
\hline$A$ & stoichiometric coefficients of reactants & $\delta$ & Kronecker delta \\
\hline$B$ & stoichiometric coefficients of products & $\Delta$ & LES filter width \\
\hline$C$ & constant & $\varepsilon$ & dissipation rate $\left(\mathrm{m}^{2} / \mathrm{s}^{3}\right)$ \\
\hline$C_{p}$ & heat capacity at constant pressure $(\mathrm{J} / \mathrm{kg} \cdot \mathrm{K})$ & $v$ & kinematic viscosity $\left(\mathrm{m}^{2} / \mathrm{s}\right)$ \\
\hline$D$ & diameter $(\mathrm{m})$ & $\rho$ & density $\left(\mathrm{kg} / \mathrm{m}^{3}\right)$ \\
\hline$g$ & gravity acceleration $\left(\mathrm{m} / \mathrm{s}^{2}\right)$ & $\sigma$ & Stefan-Boltzmann constant \\
\hline$h$ & total enthalpy $(\mathrm{J} / \mathrm{kg})$ & $\tau$ & stress tensor $\left(\mathrm{m}^{2} / \mathrm{s}^{2}\right)$ \\
\hline$H$ & height (m) & $\phi$ & local equivalence ratio \\
\hline$k$ & kinetic energy $\left(\mathrm{m}^{2} / \mathrm{s}^{2}\right)$ & $\chi$ & reacting fraction of fine structures \\
\hline$\dot{m}$ & mass transfer rate $(1 / \mathrm{s})$ & \multicolumn{2}{|c|}{ Subscripts } \\
\hline$M W$ & mole weight $(\mathrm{g} / \mathrm{mol})$ & $c$ & chemical \\
\hline$p$ & pressure $(\mathrm{Pa})$ & $d$ & hydrodynamic \\
\hline $\operatorname{Pr}$ & Prandtl number & $f u$ & fuel \\
\hline$q$ & heat flux $\left(\mathrm{W} / \mathrm{m}^{2}\right)$ & $k$ & kinetic energy \\
\hline$r$ & stoichiometric oxygen-to-fuel ratio & $m$ & species \\
\hline$R$ & ideal gas constant $(\mathrm{J} / \mathrm{mol} \cdot \mathrm{K})$ & $\max$ & maximum \\
\hline$s$ & stoichiometric air-to-fuel ratio & $p r$ & products \\
\hline$t$ & time $(s)$ & $r$ & radiation \\
\hline$T$ & temperature $(\mathrm{K})$ & $s$ & sensible \\
\hline$u$ & velocity $(\mathrm{m} / \mathrm{s})$ & $S G S$ & sub-grid scale \\
\hline$x$ & Cartesian coordinate & st & stoichiometric \\
\hline$Y$ & mass fraction & $t$ & turbulent \\
\hline$Z$ & mixture fraction & th & thermodynamic \\
\hline Greek & & & dissipation of kinetic energy \\
\hline$\alpha$ & diffusion coefficient $\left(\mathrm{m}^{2} / \mathrm{s}\right)$ & 0 & ambient condition \\
\hline$\gamma$ & mass fraction occupied by fine structures & & \\
\hline
\end{tabular}

\section{INTRODUCTION}

Fire modeling using computational fluid dynamics (CFD) technique has been evolving for decades, and various models have been proposed for the underlying combustion chemistry. However, due to the 
assumptions made during the development of the model, there still lacks a universal model that is suitable for all combustion scenarios in different combustions systems and fires.

In the fire community, the mixture fraction based combustion model is widely used by assuming the ShvabZel'dovich formulation, irreversible infinitely fast chemistry and the Burke-Schumann flame structure [1, 2]. The probability density function (PDF) approach is often adopted to take into account the effect of turbulence on combustion. Reasonably good agreement [3] of important quantities such as temperature and velocity has been achieved according to comparison with experimental data. However, the models cannot tackle problems involving extinction, such as fire behavior under water spray system, because of the underlying assumption of 'mixed is burned'. The latest version of Fire Dynamic Simulator (FDS) from NIST has included an ad-hoc modification $[4,5]$ to the mixture fraction model to allow for local flame extinction resulting from oxygen vitiation, but it has so far received only limited applications.

Magnussen and Hjertager [6] proposed the first version of the EDC based on the eddy-break-up (EBU) model of Spalding [7, 8], assuming that the chemical reaction rate is controlled by the mixing rate of fuel and oxidizer instead of the mixing rate of unburned and burned gas in the EBU. In this version, the constant $C_{E D C}$ is calculated either by the collision mixing model [6] or by the viscous mixing model [9]. Bilger [10] suggested that the coefficient is dependent on the PDF of the mixture fraction, and one can use the mixture fraction and its deviation to calculate it [11]. Bilger's work demonstrated that its value is not strongly dependent on the shape of PDF and its average value across the flame is close to that calculated by the collision mixing model [12]. However, the average value is likely to produce large errors at the fuel side where more accurate computational procedure is desirable. Subsequently, Magnussen [13] incorporated the significance of fine structures into EDC. Chemical reactions are assumed to take place in these fine structures and the extended model is formulated in a way that both finite rate chemistry and infinite fast chemistry can be used. Magnussen [14] gave a comprehensive review of the various modified version of the EDC model and demonstrated its improved predictions on some test cases. However, there still exist some limitations about the treatment of the reacting fraction within the fine structures, which is addressed in the present study.

The EDC model has been widely used in fire applications. Cox [15] applied JASMINE to model enclosure fires with forced and natural ventilations using the EDC for validation purpose and achieved reasonably good agreement with experimental data. Hutanen [16] performed fire simulations in a turbine hall using the PHOENICS code. Adiga [17] simulated a $0.25 \mathrm{~m} 28 \mathrm{~kW}$ turbulent methane fire and found that the centerline properties such as mean temperature, axial velocity and entrainment behaviors are generally well reproduced by the EDC. Wang [18] presented results on steady-state turbulent burning along a vertical rectangular channel in which a buoyancy-induced draft develops. The predictions of temperature, velocity and turbulent quantities were in good agreement with the experimental data. Novozhilov [19] tracked fire spread of solid materials subjected to constant radiant heat flux and simulated the extinguishment by water sprays. The predictions agreed well with the experiment findings. Yoon [20] carried out the studies on fire extinction behavior using the EDC-based model [21] developed at Sandia National Laboratories. His predictions are encouraging on the basis of qualitative analysis. However, all the above studies were carried out in the RANS context. Although the effect of turbulence on the combustion process is taken into account, the well known limitations of the turbulence models in RANS have to some extent limited the accuracy of these predictions. Since the EDC is highly dependent on the accuracy of the predictions for fuel-air mixing, for which the LES approach is deemed to be more appropriate, its extension to the LES context should render the model more suitable to capture the fine details of the combustion process. More recently, Panjwani [22] attempted the extension of the EDC to LES and reported the instability related to the density time derivative term. The validation study for a turbulent piloted non-premixed methane/air jet flame (Sandia Flame D) found that the model constant had to be changed from 1.01 to 0.25 . The requirement of such ad-hoc adjustment for model constant poses difficulties for it to be applied to other scenarios where experimental data is not available. In a more robust approach, the constant should be computed dynamically rather than artificially specified.

In the present study, the EDC is extended to the LES context. The formula for the reacting fraction of fine structures is modified according to the Shvab-Zel'dovich formulation to improve treatment of ignition. Corrections are also proposed for the constant in the extended EDC, which is implemented into the FireFOAM code, originally developed by FM Global based on the open source CFD code OpenFOAM. The modified version of FireFOAM has then been tested for pool fire predictions. 


\section{NUMERICAL FORMULATIONS}

Most fire problems occur at low Mach numbers. To reduce the computational cost, low Mach number formulations are adopted in this study by dividing the pressure into two parts: a time-dependent thermodynamic pressure for the energy equation and a spatial and time dependent hydrodynamic pressure for the momentum equation. This pressure decoupling prevents thermodynamic effect from interfering with the pressure in the momentum equation and so it can be solved using a conventional incompressible flow approach with a pressure update. The filtered low Mach number LES formulations are listed below where the overbars and tildes stand for spatial filtering and Favre averaging, respectively.

$$
\begin{aligned}
& \frac{\partial \bar{\rho}}{\partial t}+\frac{\partial \bar{\rho} \tilde{u}_{j}}{\partial x_{j}}=0 \\
& \frac{\partial \bar{\rho} \tilde{u}_{i}}{\partial t}+\frac{\partial \bar{\rho} \tilde{u}_{i} \tilde{u}_{j}}{\partial x_{j}}=-\frac{\partial \bar{p}}{\partial x_{i}}+\frac{\partial}{\partial x_{j}}\left(\bar{\rho}\left(v+v_{t}\right)\left(\frac{\partial \tilde{u}_{i}}{\partial x_{j}}+\frac{\partial \tilde{u}_{j}}{\partial x_{i}}-\frac{2}{3} \frac{\partial \tilde{u}_{k}}{\partial x_{k}} \delta_{i j}\right)\right)+\bar{\rho} g_{i},(i, j, k=1,2,3) \\
& \frac{\partial \bar{\rho} \tilde{h}}{\partial t}+\frac{\partial \bar{\rho} \tilde{u}_{j} \tilde{h}}{\partial x_{j}}=\frac{D \bar{p}_{t h}}{D t}+\frac{\partial}{\partial x_{j}}\left(\bar{\rho}\left(\alpha+\frac{v_{t}}{\operatorname{Pr}_{t}}\right) \frac{\partial \tilde{h}}{\partial x_{j}}\right)-\nabla \cdot \dot{q}_{r}^{\prime \prime} \\
& \frac{\partial \bar{\rho} \tilde{Y}_{m}}{\partial t}+\frac{\partial \bar{\rho} \tilde{u}_{j} \tilde{Y}_{m}}{\partial x_{j}}=\frac{\partial}{\partial x_{j}}\left(\bar{\rho}\left(\alpha+\frac{v_{t}}{\operatorname{Pr}_{t}}\right) \frac{\partial \tilde{Y}_{m}}{\partial x_{j}}\right)+\tilde{w}_{m}, \quad\left(m=f u, o_{2}, c o_{2}, h_{2} o\right) \\
& \tilde{h}=\tilde{h}_{c}+\tilde{h}_{s}=\sum_{m} h_{m}^{0} \tilde{Y}_{m}+\int_{T_{0}}^{\tilde{T}} \sum_{m}\left(C p_{m}(\tau) \tilde{Y}_{m}\right) d \tau \\
& \bar{p}=\bar{p}_{t h}+\bar{p}_{d}=\bar{p}_{0}+\bar{\rho}_{g_{i}} x_{i}+\bar{p}_{d}, \quad \bar{p}_{t h}=\bar{\rho} \frac{R}{M W} \tilde{T}
\end{aligned}
$$

The filtered equations Eqs. 2-4 are closed by a one-equation sub-grid scale (SGS) model [23] which solves a transport equation for SGS kinetic energy:

$$
\begin{aligned}
& \frac{\partial k_{S G S}}{\partial t}+\frac{\partial \tilde{u}_{j} k_{S G S}}{\partial x_{j}}=\frac{\partial}{\partial x_{j}}\left(\frac{v_{t}}{\operatorname{Pr}_{t}} \frac{\partial k_{S G S}}{\partial x_{j}}\right)-\tau_{i j} \frac{\partial \tilde{u}_{i}}{\partial x_{j}}-\varepsilon_{S G S} \\
& \varepsilon_{S G S}=C_{\varepsilon} \frac{k_{S G S}^{3 / 2}}{\Delta} ; \quad v_{t}=C_{k} k_{S G S}^{1 / 2} \Delta \\
& \tau_{i j}=-2 v_{t} \bar{S}_{i j}+\frac{2}{3} k_{S G S} \delta_{i j}, \quad \bar{S}_{i j}=\frac{1}{2}\left(\frac{\partial \tilde{u}_{i}}{\partial x_{j}}+\frac{\partial \tilde{u}_{j}}{\partial x_{i}}\right)
\end{aligned}
$$

\section{Combustion Model}

The EDC is based on the assumption that in turbulent flows, chemical reactions take place only if reactants are molecularly mixed at the smallest scales. Turbulent kinetic energy is transferred from energy containing large turbulent structures down to the fine structures where the kinetic energy is dissipated into heat. The 
dissipative fine structures are intermittently distributed, and hence only a fraction of the fine structures can be burnt. Due to the intense mixing in the fine structures, the burning proportions of the fine structures can be modeled as perfectly stirred reactors (PSR). Based on these assumptions, the reaction rate in the perfectly stirred reactors can easily be modeled as the change in mass fraction through the fine structure reactors divided by the residence time of these reactors. The mean reaction rates are then modeled as the reaction rates of the burning fine structures times the mass fraction of the burning fine structures.

As the first step to the extension of the EDC, infinitely fast one step chemistry is assumed in this study, which has proved to be sufficient in a great number of fire scenarios. Consequently, oxygen or fuel will be completely consumed in the fine structure reactors, and the variation of species mass fraction in PSR will not be solved. The reaction rate in the original EDC can be expressed as:

$$
\begin{aligned}
& \tilde{w}_{m}=\left(B_{m}-A_{m}\right) \frac{M W_{m}}{M W_{f u}} \tilde{w}_{f u}, \quad \tilde{w}_{f u}=C_{E D C} \bar{\rho} \frac{\varepsilon}{k} \min \left(\tilde{Y}_{f u}, \frac{\tilde{Y}_{o_{2}}}{r}\right), \quad C_{E D C}=\frac{11.2 \chi}{1-\gamma \chi} \\
& \gamma=4.6\left(\frac{v \varepsilon}{k^{2}}\right)^{1 / 2} ; \quad \chi=\frac{\tilde{Y}_{p r} /(1+r)}{\min \left(Y_{f u}, Y_{o_{2}} / r\right)+\tilde{Y}_{p r} /(1+r)}, \quad \tilde{Y}_{p r}=1-\tilde{Y}_{f u}-\tilde{Y}_{o_{2}}-\tilde{Y}_{n_{2}}
\end{aligned}
$$

Note that $k$ and $\varepsilon$ in the above equations should be calculated by a RANS turbulence model. It is known that in LES the large-scale flow motion are explicitly calculated, while the effect of the unresolved scales or sub-grid scales on the resolved large scales are modeled using an SGS model. Based on the turbulent energy cascading concept $[13,14]$, the length scales of the fine structures are in the order of Kolmogorov's scale, which is normally much smaller than the LES filter width in fire applications. In a RANS simulation, the turbulent energy cascaded is modeled over the whole range of spectrum, while in a LES simulation the cascade is explicitly solved for the large eddies resolved by computational grids and only partial cascade in the SGSs is modeled. Therefore, $k$ and $\varepsilon$ in Eqs. 10 and 11 are replaced with SGS quantities in this study, then

$$
\begin{aligned}
& \tilde{w}_{f u}=C_{E D C} \bar{\rho} \frac{\varepsilon_{S G S}}{k_{S G S}} \min \left(\tilde{Y}_{f u}, \frac{\tilde{Y}_{o_{2}}}{r}\right) \\
& \gamma=4.6\left(\frac{v \varepsilon_{S G S}}{k_{S G S}{ }^{2}}\right)^{1 / 2}=4.6\left(C_{\varepsilon} C_{k}\right)^{1 / 2}\left(\frac{v}{v_{t}}\right)^{1 / 2}
\end{aligned}
$$

In the LES framework, turbulent viscosity might become zero due to the local laminarization during combustion [22], causing $\gamma$, the mass fraction occupied by the fine structures (physically ranging from 0 to 1) to be ill-defined, and the EDC has no meaning for such large values of $\gamma$. In consideration of this, an upper limit of $\gamma$ is set as 1 in Ref. [22]. Equation 13 can be formulated as follows with the assumption of $v=v_{t}$,

$$
\gamma=4.6\left(C_{\varepsilon} C_{k}\right)^{1 / 2}\left(\frac{v}{v_{t}}\right)^{1 / 2}=4.6\left(C_{\varepsilon} C_{k}\right)^{1 / 2}
$$

In this study, $\gamma$ is assumed to be a constant of $4.6\left(C_{\varepsilon} C_{k}\right)^{1 / 2}$, implying that mass fraction occupied by fine structures is fixed once the turbulence model coefficients are specified before simulations. The starting point is that $C_{E D C}$ is prescribed as a constant in the early version of EDC [6, 11]. In addition, this simple treatment is supposed to tackle the numerical instability in the wall region reported in Ref. [22]. 
Base on the simplification of $\gamma$, the reacting fraction of the fine structures, i.e. $\chi$, should be modified accordingly to make the prediction of $C_{E D C}$ reasonable. Moreover, there is a limitation of its original expression as described in the following section. A new formulation of $\chi$ is proposed here. Given a mixture of fuel and oxidizer at the location where the temperature is higher, the possibility of combustion taking place near this area should be larger. Therefore, it is possible to assume $\chi$ is proportional to the flame temperature of the mixture. Thus, $\chi$ can be written as $\chi=C T$, and the constant $C$ is determined in the following way. Under the assumption of infinitely fast chemistry, the flame temperature is only a linear function of the mixture fraction $Z$ as shown in Fig. 1.

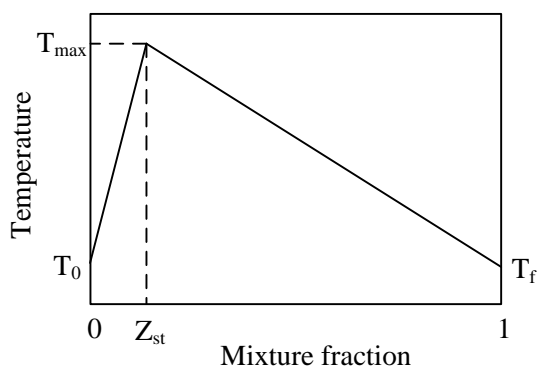

Fig. 1. Relationship between mixture fraction and flame temperature.

Therefore, if $0 \leq Z<Z_{s t}$,

$$
\frac{T-T_{0}}{T_{\max }-T_{0}}=\frac{Z}{Z_{s t}} \Rightarrow T=\frac{Z}{Z_{s t}}\left(T_{\max }-T_{0}\right)+T_{0}
$$

and if $Z_{s t} \leq Z \leq 1$,

$$
\frac{T-T_{f}}{T_{\max }-T_{f}}=\frac{1-Z}{1-Z_{s t}} \Rightarrow T=\frac{1-Z}{1-Z_{s t}}\left(T_{\max }-T_{f}\right)+T_{f}
$$

where $T_{\max }$ is adiabatic flame temperature.

Following Turns [24], the mixture fraction can be related to local equivalence ratio $\phi$,

$$
Z=\frac{\phi}{\phi+s} ; \quad Z_{s t}=\frac{1}{1+s} ; \quad \phi=\frac{s Y_{f u}}{Y_{\text {air }}} ; \quad Y_{\text {air }}=\frac{Y_{o_{2}}}{0.233}
$$

Substituting Eq. 17 into Eq. 15 and Eq. 16, if $0 \leq \phi<1, T=\frac{\phi(1+s)}{(\phi+s)}\left(T_{\max }-T_{0}\right)+T_{0}$, and if $\phi \geq 1$, $T=\frac{1+s}{\phi+s}\left(T_{\max }-T_{f}\right)+T_{f}$.

Thus, if $0 \leq \phi<1, \chi=C\left(\frac{\phi(1+s)}{(\phi+s)}\left(T_{\max }-T_{0}\right)+T_{0}\right)$, and if $\phi \geq 1, \chi=C\left(\frac{1+s}{\phi+s}\left(T_{\max }-T_{f}\right)+T_{f}\right)$.

Since theoretically complete combustion takes place at the location where $\phi=1$, it is possible to assume the reacting fraction of fine structures achieves its maximum value there, i.e. $\chi=1$. Thus, $C=1 / T_{\max }$, and 
then if $0 \leq \phi<1, \chi=\frac{\phi(1+s)}{(\phi+s)} \frac{\left(T_{\max }-T_{0}\right)}{T_{\max }}+\frac{T_{0}}{T_{\max }}$; if $\phi \geq 1, \chi=\frac{(1+s)}{(\phi+s)} \frac{\left(T_{\max }-T_{f}\right)}{T_{\max }}+\frac{T_{f}}{T_{\max }}$. Generally, $T_{0} \ll T_{\max }$ and $T_{f}<<T_{\max }$, therefore if $0 \leq \phi<1, \chi=\frac{\phi(1+s)}{(\phi+s)}$; if $\phi \geq 1, \chi=\frac{(1+s)}{(\phi+s)}$.

The detailed analysis of the effect of $\chi$ distribution on the combustion is given in the following section.

\section{Radiation Model}

Two approaches are employed in this study to take into account radiation loss. For non-sooty fires, such as the methanol fire, flame radiation is considered with the assumption of optically thin transfer between a given fluid element of flame and cold surroundings. The radiation loss term in the total enthalpy transport equation is expressed as,

$\nabla \cdot \dot{q}_{r}^{\prime \prime}=4 \sigma\left(T^{4}-T_{0}^{4}\right) \sum_{m}\left(p_{m} a_{P m}\right)$

where $p_{m}$ is the partial pressure of each specie $m$ in the fire mixture; $a_{P m}$, i.e. Planck mean absorption coefficient for each specie, is achieved from curve fits as a function of temperature based on the RADCAL program [25], as shown in Fig. 2. The detailed polynomial formulation is indicated on the TNF website.

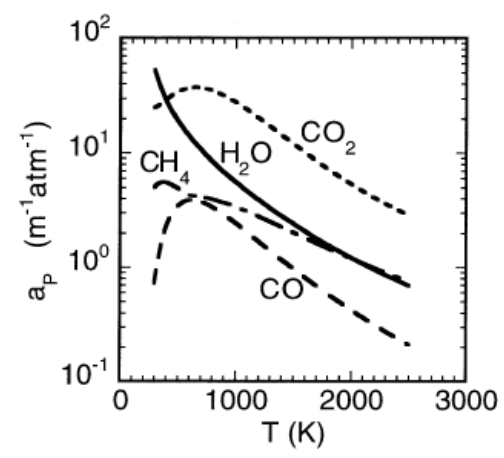

Fig. 2. Planck mean absorption coefficient as a function of temperature.

For other fires, radiation loss is approximated to be proportional to the chemical heat release rate per unit volume (i.e. $\dot{q}^{\prime \prime \prime}$ ), and described as,

$\nabla \cdot \dot{q}_{r}^{\prime \prime}=\lambda_{r} \dot{q}^{\prime \prime \prime}, \quad \dot{q}^{\prime \prime \prime}=-\frac{\partial \bar{\rho} \tilde{h}_{c}}{\partial t}-\frac{\partial \bar{\rho} \tilde{u}_{j} \tilde{h}_{c}}{\partial x_{j}}+\frac{\partial}{\partial x_{j}}\left(\bar{\rho}\left(\alpha+\frac{v_{t}}{\operatorname{Pr}_{t}}\right) \frac{\partial \tilde{h}_{c}}{\partial x_{j}}\right)$

where $\lambda_{r}$ is an empirical estimate of the local fraction of energy emitted as thermal radiation. This process is expected to bypass the soot calculation, since there is lack of a robust soot model in turbulent fire applications.

\section{NUMERICAL DESCRIPTIONS}

The governing equations are solved using a finite volume method with spatial derivatives discretized by a second order central scheme and temporal derivatives by a second order implicit scheme. The pressure is updated using a PISO scheme. The coefficients for SGS turbulent kinetic energy and its dissipation rate are set to be $C_{k}=0.04$ and $C_{\varepsilon}=0.9$, respectively; and the Prandtl number is 0.5 .

Two fire scenarios are considered in this study. One is a $30.5 \mathrm{~cm}$ diameter methanol pool fire by Weckman and Strong [26], referred to as 'Scenario 1'. In the experiment, the burner was mounted on a traversing stand that allows radial and axial traverses of the fire flow field to obtain velocity and temperature 
measurements from the centerline to the edge of the fire. On this basis, a cylindrical computational domain with the size of $90 \mathrm{~cm}$ in diameter, $180 \mathrm{~cm}$ in height was set to ensure that influence of the outflow boundaries is negligible. Non-uniform meshes were employed with grid points clustered around the burner centre and their size gradually increased in the radial and vertical directions. The number of grid points across the burner was 48 (coarse) or 90 (fine). Both are more than that used in the finest resolution case by Wen et al. [27] for the same scenario. Two types of boundary conditions were used in the calculations: free boundary conditions on the surface of the open domain and prescribed mass flow rate profiles at the fuel exit surface. The methanol feeding rate is $1.07 \mathrm{~g} / \mathrm{s}$ giving $24.6 \mathrm{~kW}$ heat release rate. The inlet temperature is $350 \mathrm{~K}$, slightly greater than the evaporation temperature of methanol. Radiation loss is accounted for with the assumption of an optically thin flame.

The other scenario is based on the small pool fires tested by Klassen and Gore [28], denoted as 'Scenario 2'. Two fire tests, including a $7.1 \mathrm{~cm}$ diameter methane fire and a $30 \mathrm{~cm}$ diameter heptane fire, are considered. Comparing to numerical studies performed by Xin et al. [29] for the $7.1 \mathrm{~cm}$ methane fire, the size of the computational domain was prescribed as $0.2 \mathrm{~m}$ in diameter and $0.4 \mathrm{~m}$ in height with 48 (coarse) or 90 (fine) grid points across the burner. The domain is open except the fuel exit to make sure that fresh air is capable of entering the domain and then sustaining the combustion. A top-hat velocity profile of $3.14 \mathrm{~cm} / \mathrm{s}$ is selected at the burner exit, and the total heat release rate of the fire is predicted to be $4.2 \mathrm{~kW}$ accordingly. The radiation fraction is estimated to be $10 \%$ [29]. For the $30 \mathrm{~cm}$ diameter heptane fire with heat release rate of $116 \mathrm{~kW}$, the settings for the domain and the grid structure are similar with the methanol fire mentioned above. The inlet temperature was set to $550 \mathrm{~K}$ to trigger combustion. The radiation fraction is set as $0.3[30]$.

For all the cases, the physical time is set to be long enough to ensure combustion is in quasi-steady state. The instantaneous results over a period of $8 \mathrm{~s}$ were averaged to produce the mean quantities. Grid sensitivity study is conducted for coarse and fine grid resolutions. The predicted results from these two grid resolutions vary within $5 \%$, so only the results from the fine mesh are presented in the next section for analysis and discussion.

\section{RESULTS AND DISCUSSIONS}

\section{Effect of $\chi$}

To some extent, the term $C_{E D C}$ represents how fast the reaction rate of fuel is consumed during the combustion. Figure 3 describes its distribution with $\gamma$ and $\chi$ ranging from 0 to 1 . It is seen that the value of $C_{E D C}$ increases with the increase of $\gamma$ and $\chi$. The values are almost two orders of magnitudes greater when $\gamma$ and $\chi$ are close to 1 . Since $\gamma$ is assumed to be a constant in this study, it appears to be evident that the distribution of $\chi$ will have a significant effect on the reaction rate.

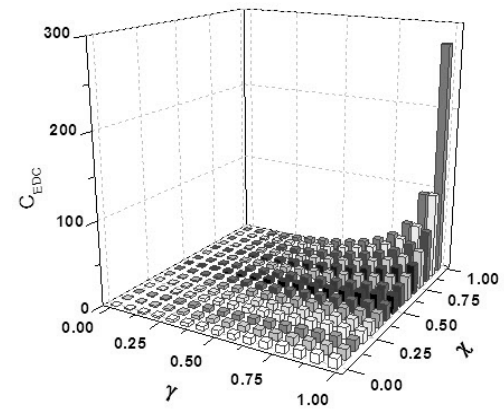

Fig. 3. $C_{E D C}$ as a function of $\gamma$ and $\chi$.

Taking the $7.1 \mathrm{~cm}$ methane fire case for example, the calculated transient and average distributions of $\chi$ and temperature across the burner centre using the original formulation of $\chi$ are shown in Fig. 4. It seems to be unreasonable that the value of $\chi$ near the fire source is close to 0 , while it is around 1 in some non-reaction area in Fig. 4. $Y_{p r}$ tends to remain 0 near the burner exit, which is because the fact that no combustion will take place if the small value is not added to $\chi$ initially, causing the chemical reaction to be frozen in this area. This is thought to be the main reason why no temperature increase as shown in the respective region 
in Fig. 4. In the far field, $Y_{f u}$ becomes 0 , and the calculated value of $\chi$ will be 1 as long as $Y_{p r}$ is not equal to 0 .

Figure 5 describes the calculated $\chi$ and temperature profiles with the new expression of $\chi$. It is indicated that the larger value of $\chi$ occurs where the flame temperature is predicted to be higher, implying that the larger reaction rate gives rise to the higher temperature according to the relationship between $C_{E D C}$ and $\chi$. In fact, this is the fundamental basis of the proposed modification. Unlike the above predictions with the original EDC model, the flame is anchored to the burner which is consistent with experimental observations [29].

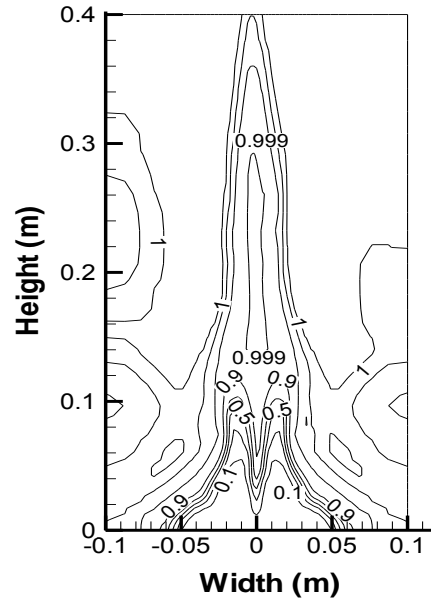

(a)

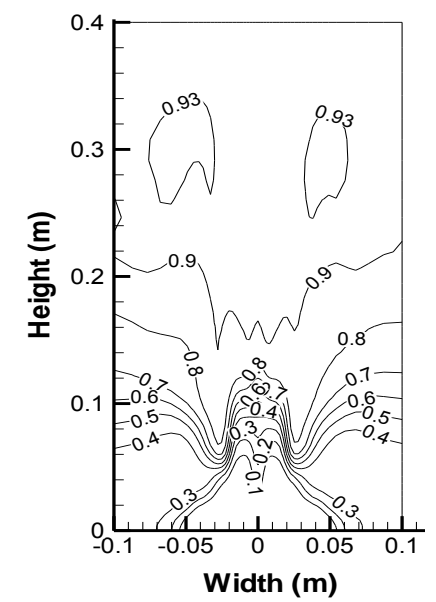

(b)

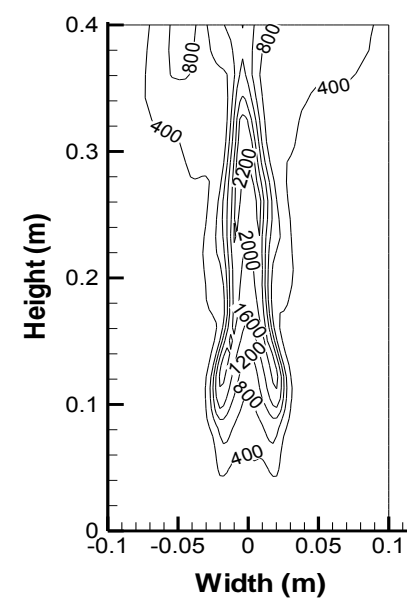

(c)

Fig. 4. Profiles for $\chi$ and temperature using the original formulation of $\chi$ : (a) transient $\chi$; (b) average $\chi$; (c) transient temperature $(\mathrm{K})$.

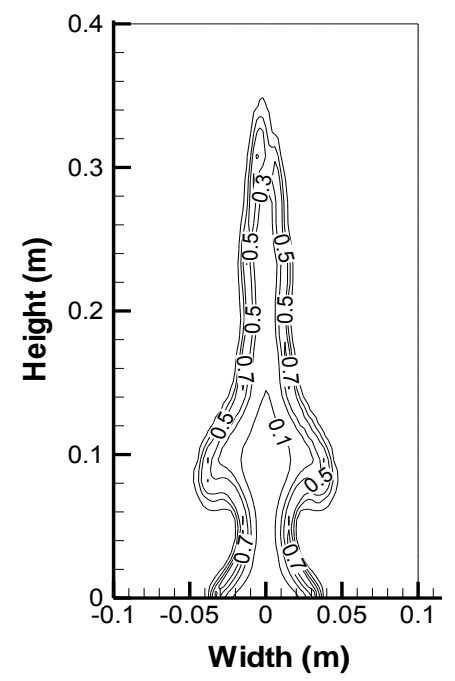

(a)

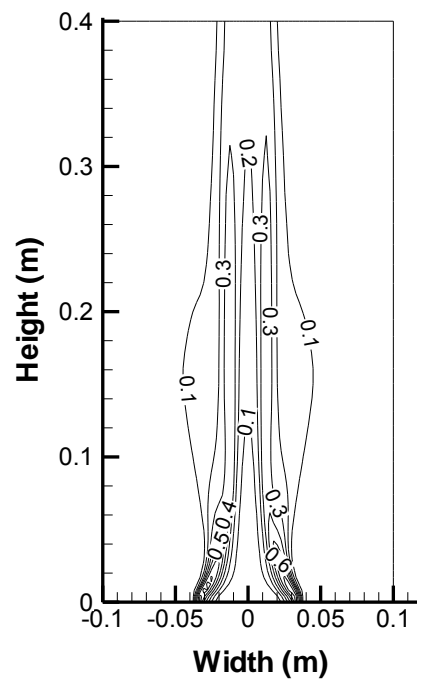

(b)

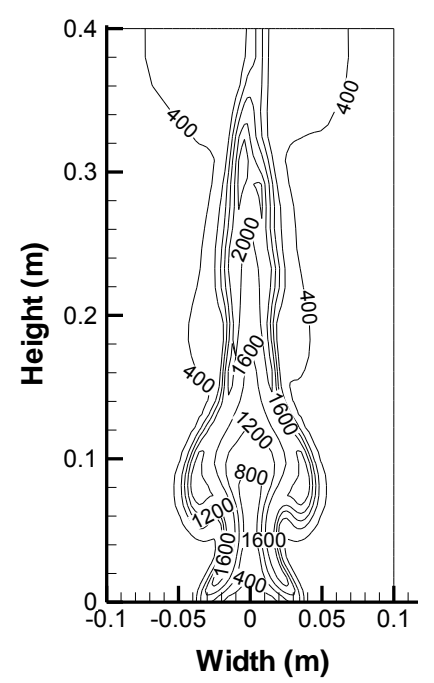

(c)

Fig. 5. Profiles for $\chi$ and temperature using the modified formulation of $\chi$ : (a) transient $\chi$; (b) average $\chi$; (c) transient temperature $(\mathrm{K})$.

\section{Scenario 1}

A comparison of the mean temperature at different heights $(6,10,18$, and $30 \mathrm{~cm})$ predicted by the EDC, the M-EDC and the experimental data is presented in Fig. 6. It is shown that the predictions from M-EDC agree well with the temperature measurements, while the original EDC under-predicts the temperature near the fire centerline. The maximum temperature difference due to the modification of $\chi$ is around $400 \mathrm{~K}$ at the height of $6 \mathrm{~cm}$ above the burner. The basis of the EDC is that the rate of combustion is linked to the 
predicted level of turbulence. Immediately above the burner surface, the turbulence level is extremely low or the flow is even laminar. This is thought to be the main reason why the original EDC significantly under-predicts the temperature there. As such, it is thought that the expression for $\chi$ needs to be amended to couple the simplification of $\gamma$. With the increase of height, the predicted temperatures by the EDC become closer to the experimental data. As the height increases, more air is entrained into the flame. Correspondingly more large eddies are produced. At some point, large eddies break up into smaller eddies, and the flow becomes more turbulent and in better agreement with the EDC concept.

Figure 7 depicts the predicted and measured temperature fluctuation profiles. For both M-EDC and EDC, the contour lines cluster near the burner rim at radial positions between $10 \mathrm{~cm}$ to $14 \mathrm{~cm}$, which is consistent with the experimental finding [26]. The big discrepancy occurs in the region near the centerline of the pool. The magnitude of the predicted temperature fluctuations by both the M-EDC and EDC increases gradually along the centerline with the increase of height. However, the predictions of the M-EDC are reasonably close to the measured values.
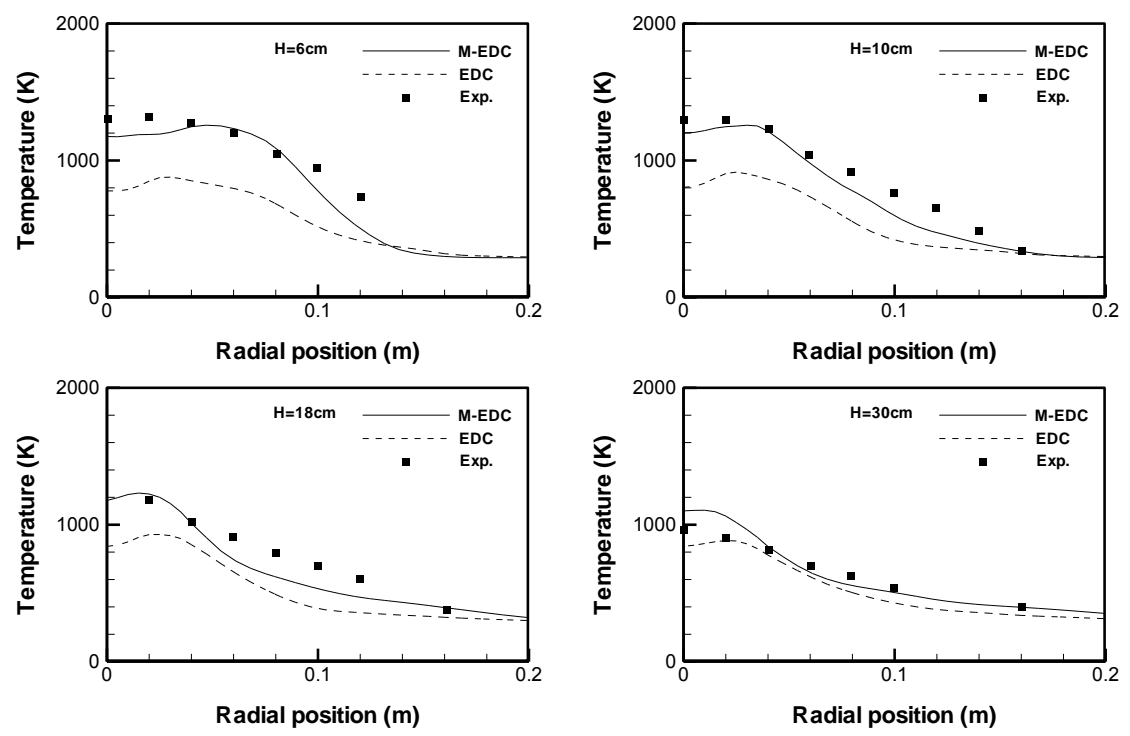

Fig. 6. Comparison of predicted and mearsured mean temperature at different locations.

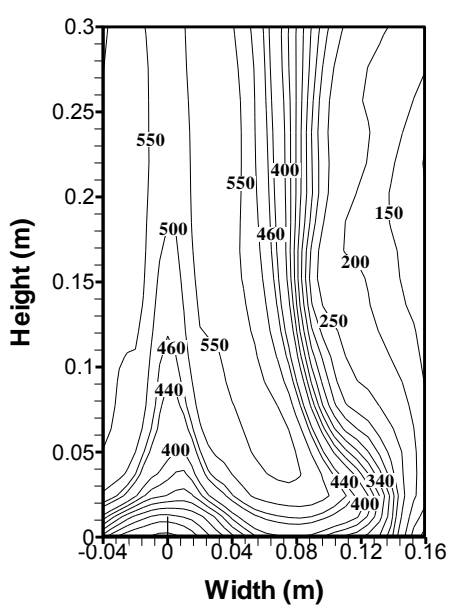

(a)

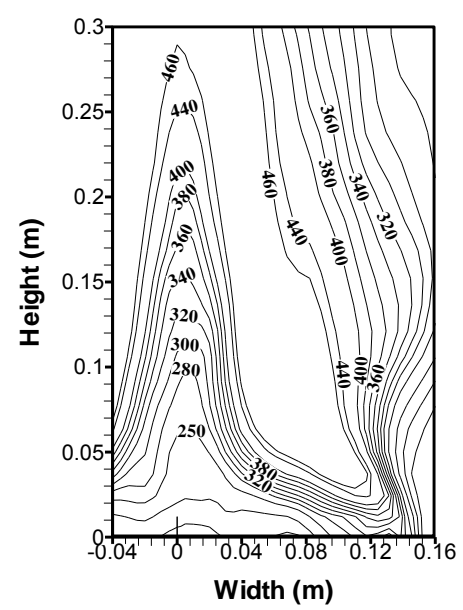

(b)

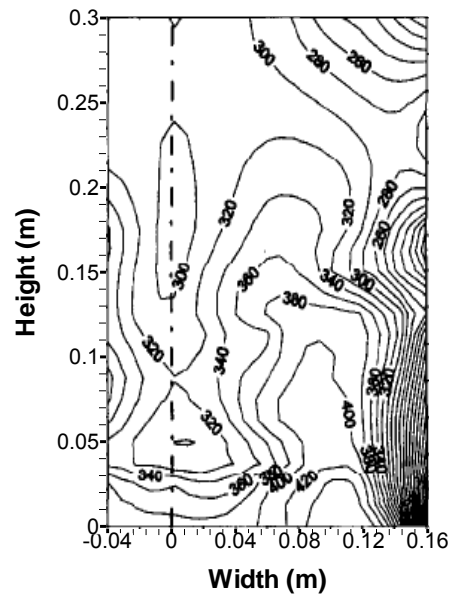

(c)

Fig. 7. Comparison of predicted and mearsured mean temperature fluctuation profiles: (a) EDC; (b) MEDC; (c) experiment. 
The mean axial velocity at different locations is presented in Fig. 8. Overall, the agreement between MEDC and the measurement is qualitatively good. The EDC under-estimates the magnitude of axial velocity, but the difference from the experimental data is not as considerable as that for the mean temperature. Figure 9 represents the comparison between the predicted and measured radial velocity distributions. It can be seen that the M-EDC and EDC give a similar magnitude of radial velocity, which is in reasonable agreement with the experimental data.
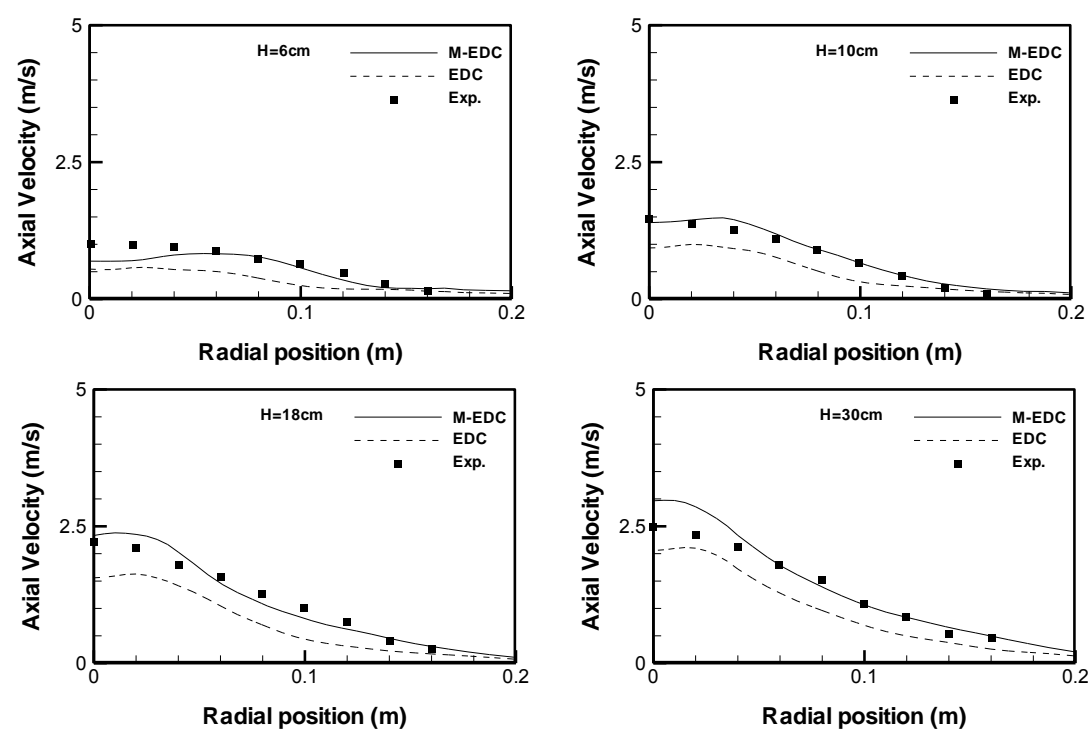

Fig. 8. Comparison of predicted and mearsured mean axial velocity at different locations.

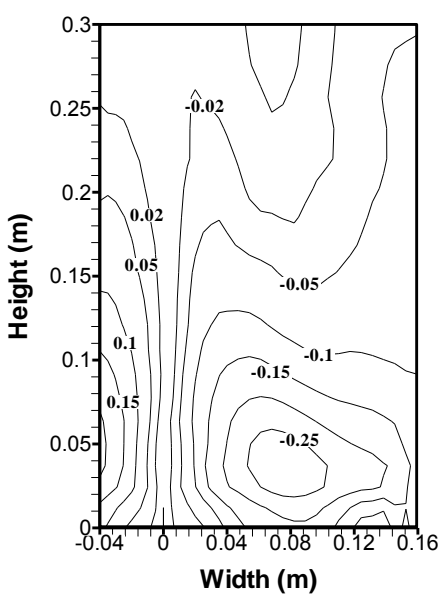

(a)

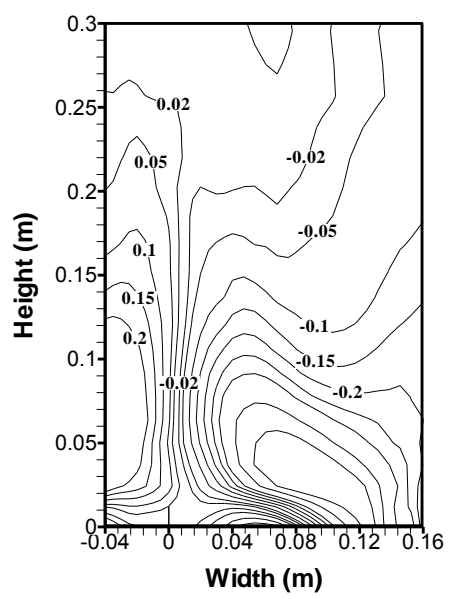

(b)

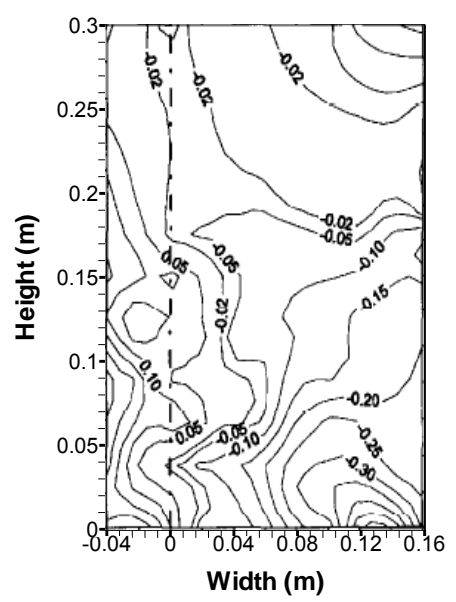

(c)

Fig. 9. Comparison of predicted and mearsured mean radial velocity profiles: (a) EDC; (b) M-EDC; (c) experiment

\section{Scenario 2}

Figure 10 shows the comparison of the predicted and measured temperature and velocity at different vertical planes for the $7.1 \mathrm{~cm}$ methane fire. It is seen that the EDC fails to predict the temperature profiles close to the burner. No temperature rise is captured below $H / D=0.14$ while the measured temperature is around $1800 \mathrm{~K}$. This is thought to be due to the ill definition of $\chi$ mentioned above. With the increase in height, the discrepancy between the calculated temperature using EDC and the experimental data decreases due to intensification of turbulence. The M-EDC is capable of predicting qualitatively similar temperature 
profiles in comparison with the experiment even near the burner surface. However, it still under-predicts the magnitude of the temperature by around $600 \mathrm{~K}$ at $H / D=0.07$ and $400 \mathrm{~K}$ at $H / D=0.14$. This is thought to be related to the fundamental assumption of mixing controlled combustion. The predictions of the MEDC are in reasonably good agreement with the measurements at $H / D=0.7$ and $H / D=1.41$.

As expected, the EDC under-predicts the values of vertical velocity and horizontal velocity for the $7.1 \mathrm{~cm}$ methane fire. For the M-EDC, the predicted vertical velocities are in reasonably good agreement with the measurements. The flame induced vertical velocity gradually increases from $H / D=0.42$ to $H / D=0.85$ due to the heat release. Although the average peak values of the top profiles are well captured, some discrepancies exist in the local values. The predicted lateral profiles are symmetric about the centre line of the burner while the measured profiles are asymmetric. The extent of the asymmetry in the experimental data suggests that this was more likely caused by a slightly windy test condition rather than due to the chaotic nature of the turbulent fire. The calculated inward horizontal velocities, which are induced by the large-scale vortices, are in very good agreement with the data. The predicted peak values and their locations also agree well with the measurements.
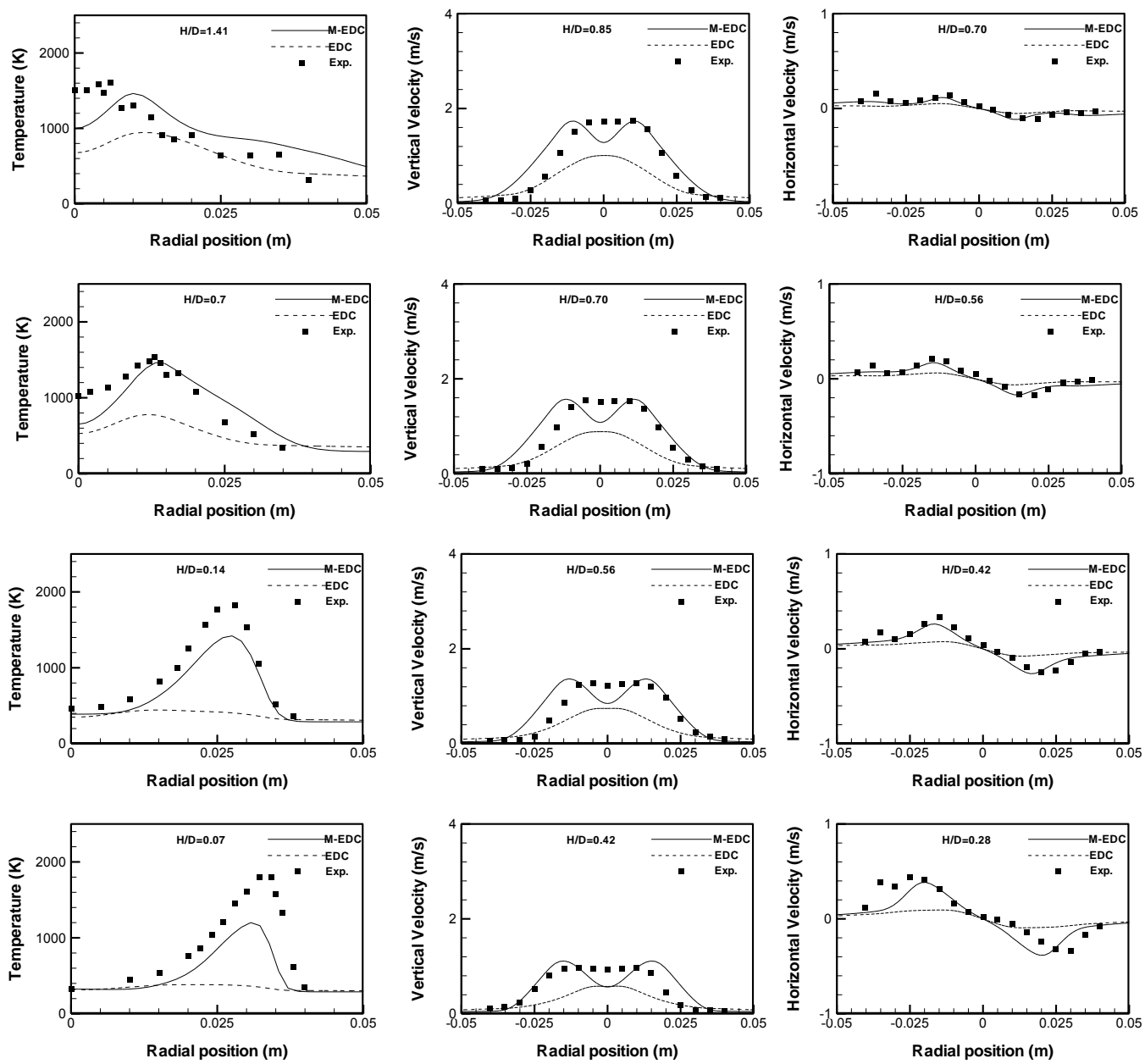

Fig. 10. Comparison of predicted and mearsured mean temperature and velocity at different locations for $7.1 \mathrm{~cm}$ methane fire.

Figure 11 plots the comparison of the predicted mean temperature and its fluctuation by the M-EDC with the measurement for the $30 \mathrm{~cm}$ heptane fire. It is observed that with an increase in height, the flame shifts inwards due to the large-scale vortices which bring fresh air into the core region. Meanwhile, the flame also expands in the radial direction and the maximum temperature decreases gradually due to turbulence enhanced mixing with ambient air. In this case, the M-EDC over-predicts the temperature profiles at three 
different heights. One possible reason is that the radiation loss is approximated to be linearly proportional to the local heat release rate as the current study does not include a soot model. It is known that heptane is indeed a very sooty fuel. The relatively larger proportion of soot in the fire plume is expected to increase the radiation loss and reduce the flame temperature. Overall, the predicted temperature fluctuations are in reasonably good agreement with the measurements.
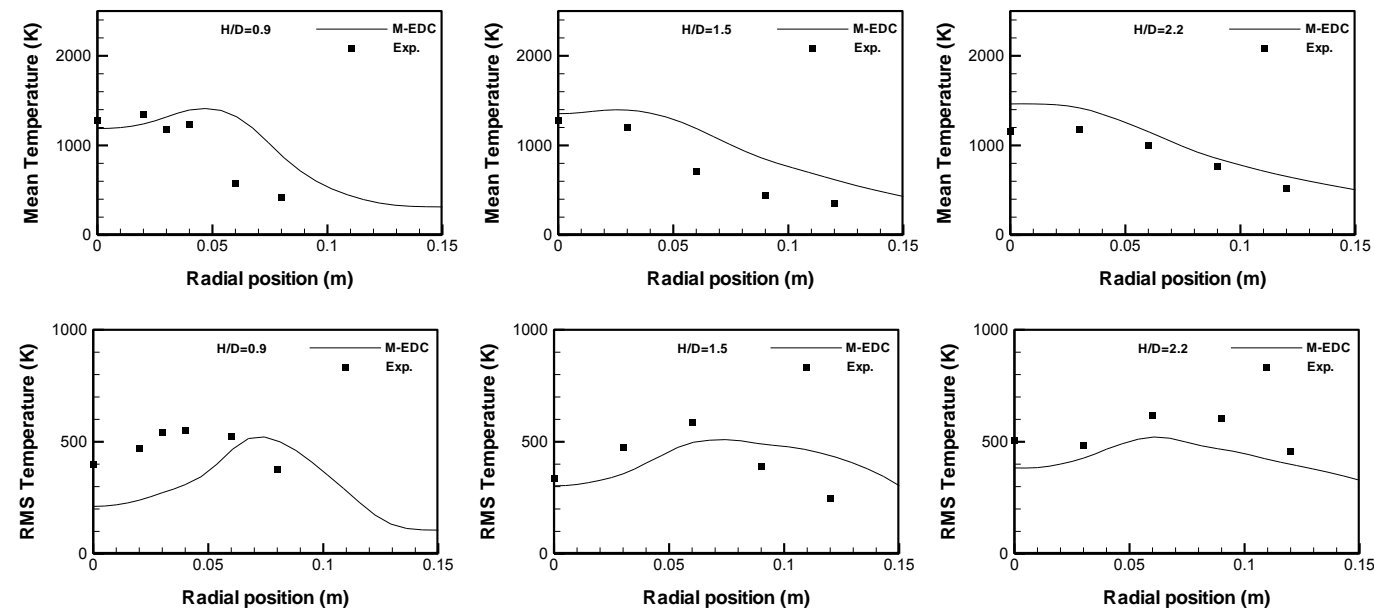

Fig. 11. Comparison of predicted and mearsured mean temperature and its fluctuations at different locations for $30 \mathrm{~cm}$ heptane fire.

\section{CONCLUSIONS}

The EDC combustion model has been extended to the LES context. The mass fraction occupied by the fine structures has been assumed to be a constant based on the SGS turbulence modeling. A more appropriate formula has been proposed to account for the reaction rate. The modified EDC has been implemented in the FireFOAM solver. The predictions from the M-EDC for a $7.1 \mathrm{~cm}$ methane fire, $30 \mathrm{~cm}$ heptane fire and $30.5 \mathrm{~cm}$ methanol fire are found to be in reasonably good agreement with the mearsurements. However, the M-EDC still under-predicts the temperatures very close to the burner surface although it has certainly shown considerable improvement over the original EDC, which was seen to under-predict all the important quantities for a much larger regions above the burner. The predicted flames of the $7.1 \mathrm{~cm}$ methane fire by the original EDC are seen to be lifted from the burner, which is inconsistent with experiment observation. It should be mentioned that while an appropriate soot model is still being sought to couple with the M-EDC, for the sooty heptane fire, radiation loss was treated as a fixed percentage of the total heat release. This simplification may have also contributed to the discrepancies. Overall, the results have demonstrated the potential of the M-EDC for fire simulations. Its computational efficiency would lend it particularly useful for the predictions of large-scale fires and fire suppression.

\section{ACKNOWLEDGEMENT}

The authors gratefully acknowledge financial and technical support from FM Global. Dr Yi Wang from FM Global, in particular, has been of great help in providing technical guidance on the source code development and application of FireFOAM.

\section{REFERENCES}

[1] Poinsot, T. and Veynante, D., Theoretical and Numerical Combustion, R.T. Edward, Philadelphia, 2005, p. 93.

[2] Williams, F.A., Combustion Theory, The Benjamin/Cummings Publishing Company, California, 1985, p. 75. 
[3] Wang, Y., Chatterjee, P. and de Ris, J.L., (2011) Large Eddy Simulation of Fire Plumes, Proceedings of the Combustion Institute 33: 2473-2480, http://dx.doi.org/10.1016/j.proci.2010.07.031

[4] McGrattan, K., Hostikka, S., Floyd, J., Baum, H. and Rehm, R., "Fire Dynamics Simulator (Version 5): Technical Reference Guide," National Institute of Standards and Technology Report NIST SP 1018-5, Gaithersburg, MD, 2007, 1-94 p.

[5] Floyd, J.E. and McGrattan, K.B., (2009) Extending the Mixture Fraction Concept to Address under-Ventilated Fires, Fire Safety Journal 44: 291-300, http://dx.doi.org/10.1016/j.firesaf.2008.07.002

[6] Magnussen, B. and Hjertager, B., (1976) On Mathematical Modeling of Turbulent Combustion with Special Emphasis on Soot Formation and Combustion, Symposium (International) on Combustion 16: 719-729, http://dx.doi.org/10.1016/S0082-0784(77)80366-4

[7] Spalding, D.B., (1971) Mixing and Chemical Reaction in Steady Confined Turbulent Flames, Symposium (International) on Combustion 13: 649-657, http://dx.doi.org/10.1016/s00820784(71)80067-x

[8] Spalding, D., (1976) Development of the Eddy-Break-up Model of Turbulent Combustion, Symposium (International) on Combustion 16: 1657-1663, http://dx.doi.org/10.1016/S0082$\underline{0784(77) 80444-X}$

[9] Magnussen, B.F., Hjertager, B.H., Olsen, J.G. and Bhaduri, D., (1979) Effects of Turbulent Structure and Local Concentrations on Soot Formation and Combustion in $\mathrm{C}_{2} \mathrm{H}_{2}$ Diffusion Flames, Symposium (International) on Combustion 17: 1383-1393, http://dx.doi.org/10.1016/s00820784(79)80130-7

[10] Bilger, R., (1989) Turbulent Diffusion Flames, Annual Review of Fluid Mechanics 21: 101-135, http://dx.doi.org/10.1146/annurev.fl.21.010189.000533

[11] Brizuela, E. and Bilger, R., (1996) On the Eddy Break-up Coefficient, Combustion and Flame 104: 208-212, http://dx.doi.org/10.1016/0010-2180(95)00205-7

[12] Novozhilov, V., (2001) Computational Fluid Dynamics Modeling of Compartment Fires, Progress in Energy and Combustion Science 27: 611-666, http://dx.doi.org/10.1016/s0360-1285(01)00005$\underline{3}$

[13] Ertesvåg, I.S. and Magnussen, B.F., (2000) The Eddy Dissipation Turbulence Energy Cascade Model, Combustion Science and Technology 159: 213 - 235, http://dx.doi.org/10.1080/00102200008935784

[14] Magnussen, B.F., "The Eddy Dissipation Concept: A Bridge between Science and Technology," Thematic Conference on Computational Combustion, The European Community on Computational Methods in Applied Sciences, 2005, pp. 1-25.

[15] Cox, G. and Kumar, S., (1987) Field Modelling of Fire in Forced Ventilated Enclosures, Combustion Science and Technology 52: 7 - 23, http://dx.doi.org/10.1080/00102208708952565

[16] Huhtanen, R., 1989. Numerical Fire Modelling of a Turbine Hall. Fire Safety Science 2: 771-779. http://dx.doi.org/10.3801/IAFSS.FSS.2-771

[17] Adiga, K.C., Ramaker, D.E., Tatem, P.A. and Williams, F.W., (1990) Numerical Predictions for a Simulated Methane Fire, Fire Safety Journal 16: 443-458, http://dx.doi.org/10.1016/0379$\underline{7112(90) 90021-6}$

[18] Wang, H.Y. and Joulain, P., (1996) Three-Dimensional Modeling for Prediction of Wall Fires with Buoyancy-Induced Flow Along a Vertical Rectangular Channel, Combustion and Flame 105: 391-406, http://dx.doi.org/10.1016/0010-2180(95)00212-x

[19] Novozhilov, V., Harvie, D.J.E., Kent, J.H., Apte, V.B. and Pearson, D., (1997) A Computational Fluid Dynamics Study of Wood Fire Extinguishment by Water Sprinkler, Fire Safety Journal 29: 259-282, http://dx.doi.org/10.1016/s0379-7112(97)00027-1 
[20] Yoon, S.S., Figueroa, V., Brown, a.L. and Blanchat, T.K., (2009) Experiments and Modeling of Large-Scale Benchmark Enclosure Fire Suppression, Journal of Fire Sciences 28: 109-139, http://dx.doi.org/10.1177/0734904109104503

[21] DesJardin, P. and Gritzo, L., "A Dilute Spray Model for Fire Simulations: Formulation, Usage and Benchmark Problems," Sandia National Laboratories Report SAND 2002-3419, Albuquerque, NM, 2002, 1-89 p.

[22] Panjwani, B., Ertesvag, I.S., Rian, K.E. and Gruber, A., "Sub-Grid Combustion Modeling for Large Eddy Simulation (LES) of Turbulent Combustion Using Eddy Dissipation Concept," Fifth European Conference on Computational Fluid Dynamics, The European Community on Computational Methods in Applied Sciences, 2010, pp. 1-19.

[23] Menon, S., Yeung, P.K. and Kim, W.W., (1996) Effect of Subgrid Models on the Computed Interscale Energy Transfer in Isotropic Turbulence, Computers and Fluids 25: 165-180, http://dx.doi.org/10.1016/0045-7930(95)00036-4

[24] Turns, S., An Introduction to Combustion-Concepts and Applications, McGraw-Hill, New York, 2000, p. 245.

[25] Grosshandler, W., "Radcal: A Narrow-Band Model for Radiation Calculations in a Combustion Environment," National Institute of Standards and Technology Report NIST Technical Note 1402, Gaithersburg, MD, 1993, 1-52 p.

[26] Weckman, E.J. and Strong, A.B., (1996) Experimental Investigation of the Turbulence Structure of Medium-Scale Methanol Pool Fires, Combustion and Flame 105: 245-266, http://dx.doi.org/10.1016/0010-2180(95)00103-4

[27] Wen, J.X., Kang, K., Donchev, T. and Karwatzki, J.M., (2007) Validation of Fds for the Prediction of Medium-Scale Pool Fires, Fire Safety Journal 42: 127-138, http://dx.doi.org/10.1016/j.firesaf.2006.08.007

[28] Klassen, M. and Gore, J., "Structure and Radiation Properties of Pool Fires: Final Report," National Institute of Standards and Technology Report NIST-GCR-94-651, Gaithersburg, MD, 1992, 1-153 p.

[29] Xin, Y., Gore, J., McGrattan, K., Rehm, R. and Baum, H., (2005) Fire Dynamics Simulation of a Turbulent Buoyant Flame Using a Mixture-Fraction-Based Combustion Model, Combustion and Flame 141: 329-335, http://dx.doi.org/10.1016/j.combustflame.2004.07.001

[30] Drysdale, D., An Introduction to Fire Dynamics, Wiley, Chichester, 1999, p. 146. 